\title{
HIPK2 inhibits cell metastasis and improves chemosensitivity in esophageal squamous cell carcinoma
}

\author{
ZHEN ZHANG $^{1}$, PENGHAO WEN ${ }^{2}$, FANGFANG LI ${ }^{1}$, CHUANSHAN YAO $^{1}$, \\ TONGFU WANG ${ }^{1}$, BING LIANG ${ }^{1}$, QINGLE YANG $^{1}$, LEI MA $^{1}$ and LIMIN HE ${ }^{1}$ \\ ${ }^{1}$ Department of Clinical Oncology, The First People's Hospital of Nanyang; ${ }^{2}$ Department of Clinical Oncology, \\ The Affiliated Nanshi Hospital of Henan University, Nanyang, Henan 473000, P.R. China
}

Received March 9, 2017; Accepted September 22, 2017

DOI: $10.3892 / \mathrm{etm} .2017 .5468$

\begin{abstract}
Esophageal squamous cell carcinoma (ESCC) is one of the most aggressive and lethal malignancies worldwide. At present, the underlying mechanisms of ESCC development and progression are poorly understood. Previous studies have demonstrated that homeodomain-interacting protein kinase-2 (HIPK2) serves an important role in cancer biology, particularly in proliferation and metastasis. However, the role of HIPK2 in ESCC cells remains unclear. In the current study, the expression of HIPK2 in ESCC specimens, adjacent non-cancerous tissues and cell lines was assessed using reverse transcription-quantitative polymerase chain reaction (RT-qPCR). The effects of HIPK2 on cell metastasis, epithelial-mesenchymal transition (EMT) and proliferation were studied using a Transwell assay, RT-qPCR and a Cell Counting Kit-8 assay, respectively. The results indicated that HIPK2 expression was downregulated in ESCC specimens and cell lines, and HIPK2 expression was associated with tumor invasion and lymph node metastasis. Functional studies demonstrated that HIPK2 overexpression inhibited cell metastasis and EMT. Furthermore, HIPK2 overexpression suppressed cell viability during cisplatin treatment. These results suggest that HIPK2 serves an important role in regulating metastasis and the chemosensitivity of ESCC cells, implicating the potential application of HIPK2 in ESCC therapy.
\end{abstract}

\section{Introduction}

Esophageal squamous cell carcinoma (ESCC) is one of the most aggressive and lethal malignancies worldwide (1). Nutritional deficiencies, nitrosamine-rich or mycotoxin-contaminated foods and low socioeconomic status are likely to contribute

Correspondence to: Professor Limin He or Dr Lei Ma, Department of Clinical Oncology, The First People's Hospital of Nanyang, 12 Renmin Road, Nanyang, Henan 473000, P.R. China

E-mail: helimin01ch@163.com

E-mail: malei1225ch@163.com

Key words: esophageal squamous cell carcinoma, homeodomaininteracting protein kinase-2, metastasis, chemoresistance to ESCC $(1,2)$. It has been reported that heavy smoking and alcohol consumption are key environmental risk factors for ESCC $(3,4)$. Dysphagia is the most common symptom of ESCC. Although surgery is considered to be the most effective treatment for ESCC in terms of locoregional control and long-term survival, recurrence and metastases to the liver occur in the majority of cases following complete resection, resulting in a $15-25 \%$ five-year survival rate in patients with ESCC (5). Therefore, the identification of molecular mechanisms that pinpoint biologically aggressive tumors is critical for the effective management of ESCC.

Homeodomain-interacting protein kinase-2 (HIPK2) is a member of an evolutionary conserved family of serine/threonine kinases and is considered to be a tumor suppressor that modulates a number of basic cellular processes, including apoptosis, proliferation, differentiation and metastasis (6-9). It has previously been demonstrated that HIPK2 mediates apoptosis and epithelial-mesenchymal transition (EMT) of renal tubular epithelial cells contributing to fibrosis, implying that it may be a potential target for anti-fibrosis therapy (10). HIPK2 has also been demonstrated to activate the p53 protein via direct binding and phosphorylation at serine 46 following severe DNA damage (11). Puca et al (12) demonstrated that HIPK2 is an important regulator of p53 activity in response to chemotherapeutic agent cisplatin and Lazzari et al (13) indicated that HIPK2 knockdown induces resistance to multiple anticancer agents, including doxorubicin and cisplatin. HIPK2-mediated vimentin downregulation may contribute to the inhibition of breast cancer cell invasion (14). In bladder cancer, HIPK2 inhibition promotes EMT and subsequent cell invasion, at least in part by activating Wnt signaling (15). However, the biological role and clinical significance of HIPK2 in ESCC remain largely unknown.

The present study aimed to investigate whether HIPK2 regulates metastasis and chemosensitivity in ESCC. It was identified that upregulation of HIPK2 inhibits cell metastasis and suppresses cell viability during cisplatin treatment, implicating a potential application of HIPK2 in ESCC therapy.

\section{Materials and methods}

ESCC specimens. A total of 56 paired ESCC specimens (34 males and 22 females) and adjacent non-cancerous tissues 
were collected from the Department Of Thoracic Surgery of the First People's Hospital of Nanyang (Nanyang, China) between March 2015 and February 2016. The mean age was 63.22 years (range, $44-84$ years). According to the AJCC tumor stage (16), 30 patients had stage 1-2 and 26 had stage 3-4. Samples were immediately frozen in liquid nitrogen and stored at $-80^{\circ} \mathrm{C}$. Written informed consent was obtained from all patients prior to their involvement in the current study. None of the patients had undergone preoperative anticancer therapies. The study was approved by the Ethics Committee of the First People's Hospital of Nanyang.

Cell lines and transfection. Human ESCC cell lines EC109 and EC9706 clone EC1 (EC1) and the human epithelial cell line Het-1a were maintained in RPMI-1640 (Hyclone; GE Healthcare Life Sciences, Logan, UT, USA) supplemented with $10 \%$ fetal bovine serum (FBS, Gibco; Thermo Fisher Scientific, Inc., Waltham, MA, USA) in a humidified atmosphere with $5 \% \mathrm{CO}_{2}$ at $37^{\circ} \mathrm{C}$. The cisplatin-resistant sub-line (EC109/cis) was established by continuous exposure to increasing concentrations $(0.1,0.2,0.4,0.6$ and $1 \mu \mathrm{g} / \mathrm{ml})$ cisplatin over 12 months (17). After continuous exposure to cisplatin for 2 days, the medium was replaced with a fresh cisplatin free medium until the surviving cells recovered favorably. When cells grew to the $60-70 \%$ confluency, cisplatin was added to the medium again. Each concentration was repeated six times.

The pEGFP-N1 and pEGFP-N1-HIPK2 plasmids were purchased from Shanghai GenePharma Co., Ltd. (Shanghai, China) and verified by sequencing using an ABI 3730xl automated sequencer (Applied Biosystems; Thermo Fisher Scientific, Inc.). For plasmid transfection, $3 \times 10^{5}$ cells (EC109, EC1, EC109/cis) were seeded in 6-well plates $24 \mathrm{~h}$ prior to transfection with $4 \mu \mathrm{g}$ plasmid DNA using Lipofectamine 2000 (Invitrogen; Thermo Fisher Scientific, Inc.) according to the manufacturer's protocol. Transfected cells were selected using G418 (Sigma-Aldrich; Merck KGaA, Darmstadt, Germany) 2 days after transfection to generate stably transfected monoclonal cell lines. After 14 days of screening, stable transfectants were selected for further amplification, and were then tested by reverse transcription-quantitative polymerase chain reaction (RT-qPCR) for overexpression of HIPK2.

$R T-q P C R$. Total RNA was extracted from cells (Het-1a, EC109, EC1, EC109/cis) and ESCC specimens using TRIzol according to the manufacturer's protocol (Invitrogen; Thermo Fisher Scientific, Inc.), and RT reactions were performed using a PrimeScript ${ }^{\mathrm{TM}}$ II 1st Strand cDNA Synthesis kit (Takara Biotechnology Co.,Ltd., Dalian, China) according to the manufacturer's protocol. PCR analysis was performed using $\mathrm{SYBR}^{\circledR}$ Premix Ex Taq ${ }^{\mathrm{TM}}$ II reagent (Takara Biotechnology Co., Ltd.) on the ABI 7500 Fast System (Applied Biosystems; Thermo Fisher Scientific, Inc.). The reaction protocol involved heating for $10 \mathrm{sec}$ at $95^{\circ} \mathrm{C}$, followed by 40 cycles of amplification (5 sec at $95^{\circ} \mathrm{C}$ and $30 \mathrm{sec}$ at $60^{\circ} \mathrm{C}$ ). The primer sequences (HIPK2, GAPDH, E-cadherin and N-cadherin) used are presented in Table I. GAPDH was used as the internal standard. Data analysis was performed using the $2^{-\Delta \Delta \mathrm{Cq}}$ method (18). Each sample was analyzed in triplicate.
Transwell migration and invasion assay. Transwell cell migration and Matrigel invasion assays ( $8 \mu \mathrm{m}$ pore size) were utilized to estimate the migration and invasion ability of cells (EC109 and EC1) in vitro. For the invasion assay, Matrigel (BD Biosciences, Franklin Lakes, NJ, USA) was added to the upper surface of the membrane and allowed to gel at $37^{\circ} \mathrm{C}$ for $30 \mathrm{~min}$. A total of $600 \mu \mathrm{l}$ of RPMI-1640 medium with 10\% FBS was placed in the bottom compartment of the chamber. Harvested EC109 and EC1 cells $\left(2 \times 10^{4}\right)$ in $200 \mu 1$ of serum-free RPMI 1640 medium were added into the upper compartment of the chamber. Following $24 \mathrm{~h}$ of incubation at $37^{\circ} \mathrm{C}$ in an atmosphere containing $5 \% \mathrm{CO}_{2}$, cells that had migrated or invaded to the basal side of the membrane were stained with crystal violet $(0.005 \%$, Sigma-Aldrich; Merck KGaA) for $20 \mathrm{~min}$ at room temperature and counted as the number of cells per field of view under a light microscope at x200 magnification in 10 random fields.

Cell Counting Kit(CCK)-8assay. Cells (EC109-Vector/HIPK2, EC109/cis-Vector/HIPK2) were seeded in triplicate on a 96-well plate (Corning Incorporated, Corning, NY, USA) at a density of $5 \times 10^{3}$ cells/well. Cell proliferation was assessed after 24, 48 and $72 \mathrm{~h}$ using the CCK-8 (Dojindo Molecular Technologies, Inc., Kumamoto, Japan) method according to the manufacturer's protocol.

Statistical analysis. All data are expressed as the mean \pm standard deviation. The differences between two groups were analyzed using Student's t-test. The difference between three groups was assessed by one-way analysis of variance followed by Tukey's post hoc test. All statistical analyses were performed using SPSS 17.0 software (SPSS, Inc., Chicago, IL, USA). $\mathrm{P}<0.05$ was considered to indicate a statistically significant difference.

\section{Results}

HIPK2 expression is decreased in ESCC cells. The expression of HIPK2 in 56 paired ESCC tissues was determined using RT-qPCR. The expression of HIPK2 mRNA was significantly decreased in ESCC tissues compared with their adjacent non-cancerous tissues $(\mathrm{P}<0.01$; Fig. 1A). To further evaluate the role of HIPK2 in human ESCC, the association between HIPK2 and clinical parameters, including age, sex, TNM and cancer grade, was evaluated. Decreased HIPK2 expression was significantly associated with tumor invasion $(\mathrm{P}<0.05$; Fig. 1B) and lymph node metastasis ( $\mathrm{P}<0.05$; Fig. 1C). HIPK2 was not significantly associated with other clinical characteristics, including age, gender, differentiation and tumor stage (data not shown). The expression of HIPK2 in ESCC cell lines EC109 and EC1 and esophageal epithelial cell line Het-1a was then determined. HIPK2 was significantly downregulated in EC109 and EC1 cells compared with Het-1a cells (both $\mathrm{P}<0.01$; Fig. 1D). These results indicated that HIPK2 downregulation may be associated with ESCC metastasis and serve a tumor suppressive role in ESCC.

HIPK2 overexpression inhibits cell migration and invasion in vitro. To further investigate the role of HIPK 2 in cell invasion, ESCC cells stably transfected with a control vector or HIPK2 
Table I. Primer sequences.

\begin{tabular}{llll}
\hline Gene & \multicolumn{1}{c}{ Forward (5'-3') } & \multicolumn{1}{c}{ Reverse (5'-3') } & Product size (bp) \\
\hline GAPDH & GCACCGTCAAGGCTGAGAAC & TGGTGAAGACGCCAGTGGA & 138 \\
HIPK2 & CCCGTGTACGAAGGTATGGC & AGTTGGAACTCGGCTCTATTTC & 109 \\
N-cadherin & AGCCAACCTTAACTGAGGAGT & GGCAAGTTGATTGGAGGGATG & 136 \\
E-cadherin & ATTTTTCCCTCGACACCCGAT & TCCCAGGCGTAGACCAAGA & 109
\end{tabular}

HIPK2, homeodomain-interacting protein kinase-2; N, neural; E, epithelial.
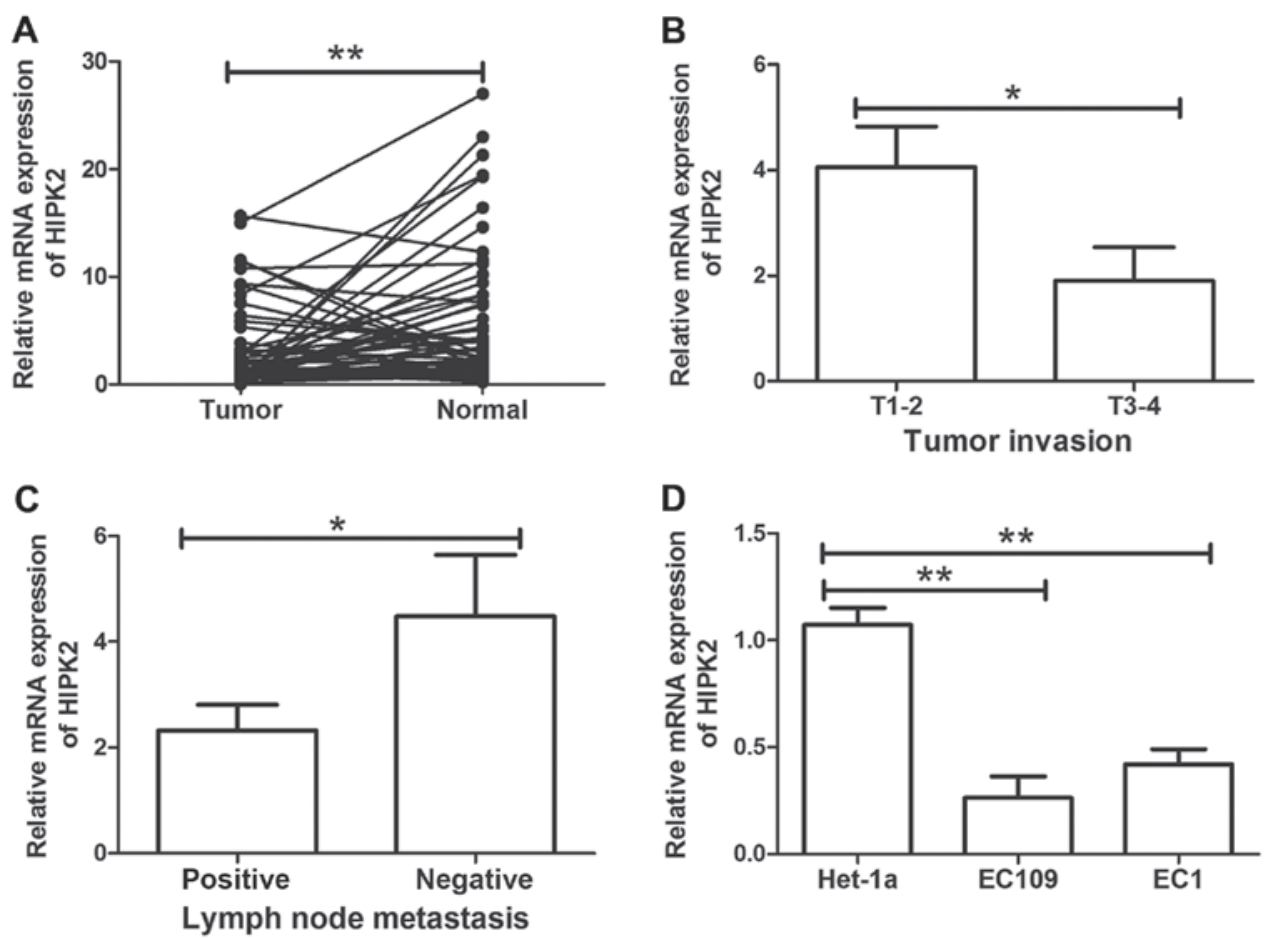

Figure 1. HIPK2 expression is downregulated in ESCC cells and associated with tumor invasion and lymph node metastasis. HIPK2 expression was assessed in (A) 56 paired ESCC specimens and adjacent non-cancerous tissues, (B) T1-2 $(n=30)$ and T3-4 $(n=26)$ groups according to the depth of tumor invasion, (C) lymph node metastasis negative and positive groups and (D) human ESCC cell lines EC109 and EC1 and human epithelial cell line Het-1a. Each test was repeated in triplicate. ${ }^{*} \mathrm{P}<0.05$ and ${ }^{* *} \mathrm{P}<0.01$. HIPK2, homeodomain-interacting protein kinase-2; ESCC, esophageal squamous cell carcinoma; $\mathrm{T}$, tumor; Normal, adjacent non-cancerous tissue.

were analyzed. The expression of HIPK2 was confirmed in EC109 (Fig. 2A) and EC1 cells (Fig. 2B) transfected with HIPK 2 or the control vector. HIPK 2 mRNA was significantly increased in EC109 and EC1 cells transfected with HIPK2 compared with cells transfected with the control vector (both $\mathrm{P}<0.001)$. In addition, HIPK2 overexpression significantly inhibited ESCC cell migration and invasion in vitro $(\mathrm{P}<0.05$; Fig. 2C and D). These results suggest that HIPK2 negatively regulates ESCC cell migration and invasion.

HIPK2 overexpression suppresses EMT. The initial stage of metastasis is dependent on the prominent biological event referred to as EMT, which is characterized by specific morphogenetic changes, loss of cell adhesion and increased cell movement (19). The mechanism by which HIPK 2 regulates ESCC cell metastasis was investigated. HIPK2 overexpression decreased the expression of the mesenchymal marker neural (N)-cadherin mRNA in EC109 (P<0.01; Fig. 3A) and EC1 cells $(\mathrm{P}<0.01$; Fig. 3B). HIPK2 overexpression significantly increased the expression of epithelial (E)-cadherin mRNA in EC109 and EC1 cells (both $\mathrm{P}<0.05$; Fig. 3C and D). These results indicate that EMT was suppressed by HIPK2 upregulation.

HIPK2 overexpression inhibits cell viability during cisplatin treatment. Cisplatin-based chemotherapy is a common regimen applied for the treatment of ESCC (20). Chemoresistance is a primary cause of treatment failure in patients with cancer (21). To clarify the molecular mechanisms underlying cisplatin resistance in ESCC cells, a cisplatin resistant subline was established. The EC109 ESCC cell line was treated with cisplatin in gradually increasing concentrations to establish a cisplatin resistant cell line (EC109/cis). HIPK2 was significantly downregulated in chemoresistant EC109/cis cells $(\mathrm{P}<0.01$; Fig. 4A), indicating that HIPK2 downregulation may be associated with cisplatin resistance in ESCC cells. The 

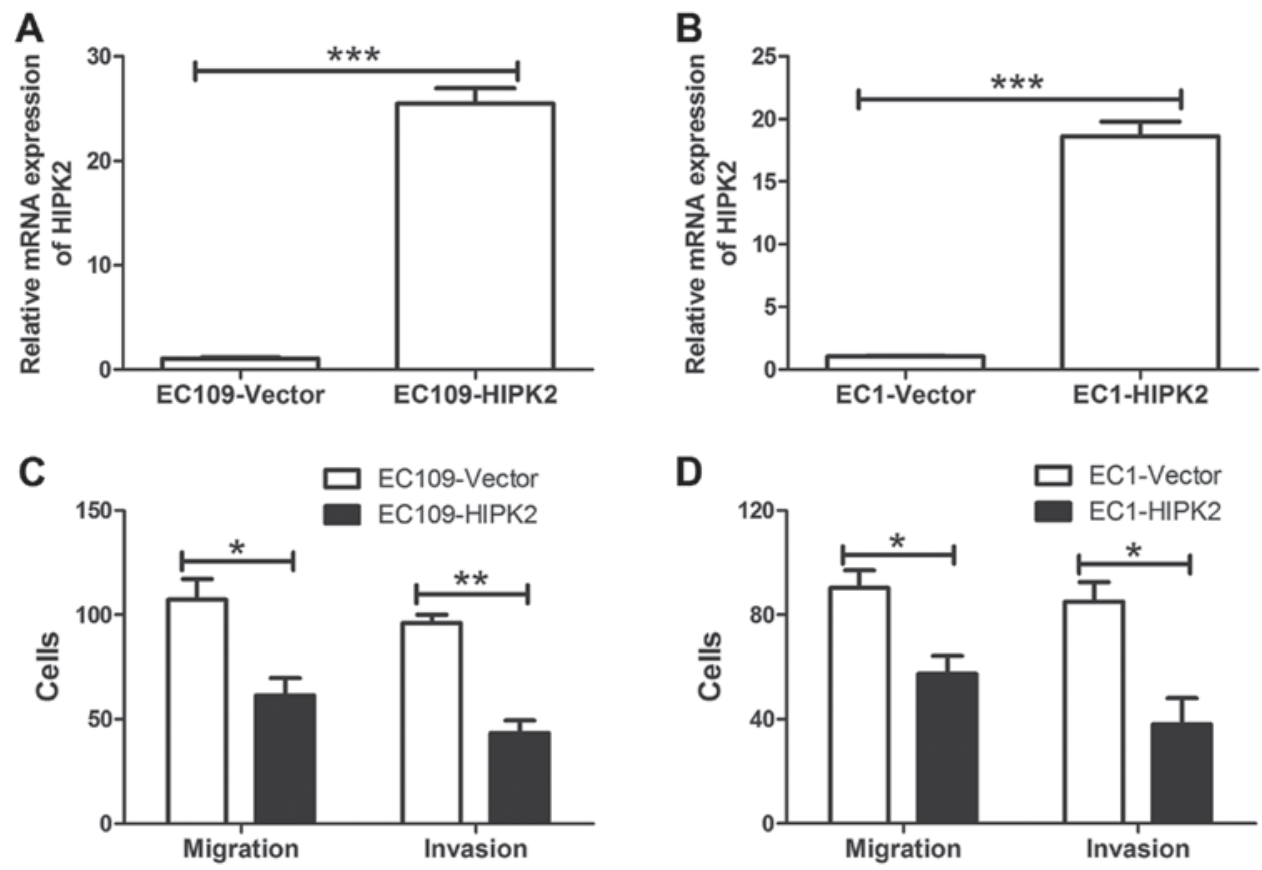

Figure 2. HIPK2 overexpression inhibits cell migration and invasion in vitro. HIPK2 expression was confirmed by reverse transcription-quantitative polymerase chain reaction in (A) EC109 and (B) EC1 cells transfected with HIPK2 and empty vector. Transwell and Matrigel migration and invasion assays were performed for (C) EC109 and (D) EC1 cells transfected with HIPK2 and empty vector. Each test was repeated in triplicate. ${ }^{*} \mathrm{P}<0.05,{ }^{* * *} \mathrm{P}<0.01$ and ${ }^{* * * *} \mathrm{P}<0.001$. HIPK2, homeodomain-interacting protein kinase-2.

A

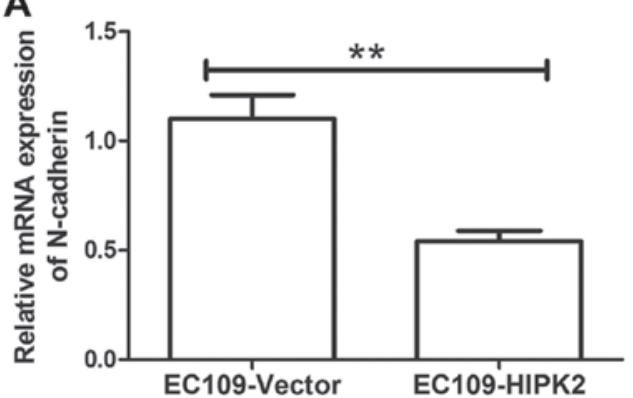

C

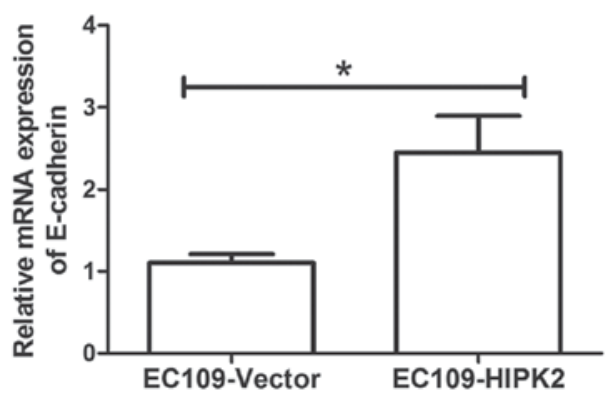

B

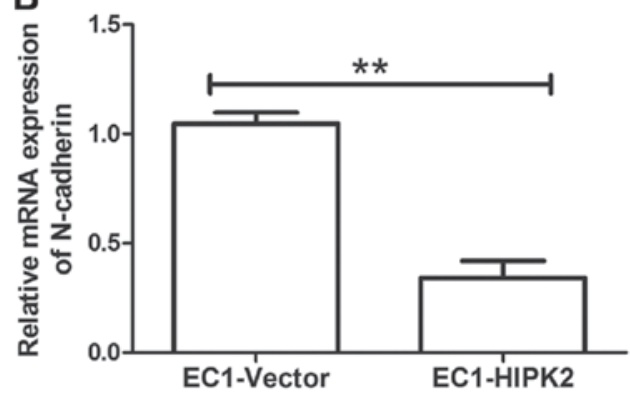

D

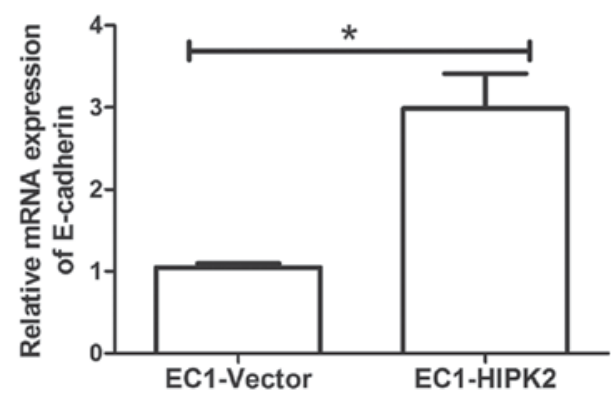

Figure 3. HIPK2 overexpression suppresses EMT. The expression of N-cadherin was investigated in (A) EC109 and (B) EC1 cells transfected with HIPK2 and empty vector. The expression of E-cadherin was investigated in (C) EC109 and (D) EC1 cells transfected with HIPK2 and empty vector. Each test was repeated in triplicate. ${ }^{*} \mathrm{P}<0.05$ and ${ }^{* *} \mathrm{P}<0.01$. HIPK2, homeodomain-interacting protein kinase-2; EMT, epithelial-mesenchymal transition; $\mathrm{N}$, neural; E, epithelial.

effect of HIPK2 on cisplatin sensitivity in ESCC cells was also investigated. HIPK2 overexpression significantly decreased EC109 cell viability compared with EC109 cells transfected with the control vector during cisplatin treatment $(\mathrm{P}<0.001$; Fig. 4B). In EC109/cis cells, cisplatin treatment caused a modest decrease in cell viability compared with EC109 cells, whereas overexpression of HIPK2 in EC109/cis cells restored the sensitivity of chemoresistant cells to cisplatin $(\mathrm{P}<0.01$; Fig. 4C and D). The overexpression of HIPK2 had no significant effect on the proliferation of EC109 cells compared with EC109/cis cells. These results suggest that HIPK2 increased the cisplatin sensitivity of ESCC cells. 

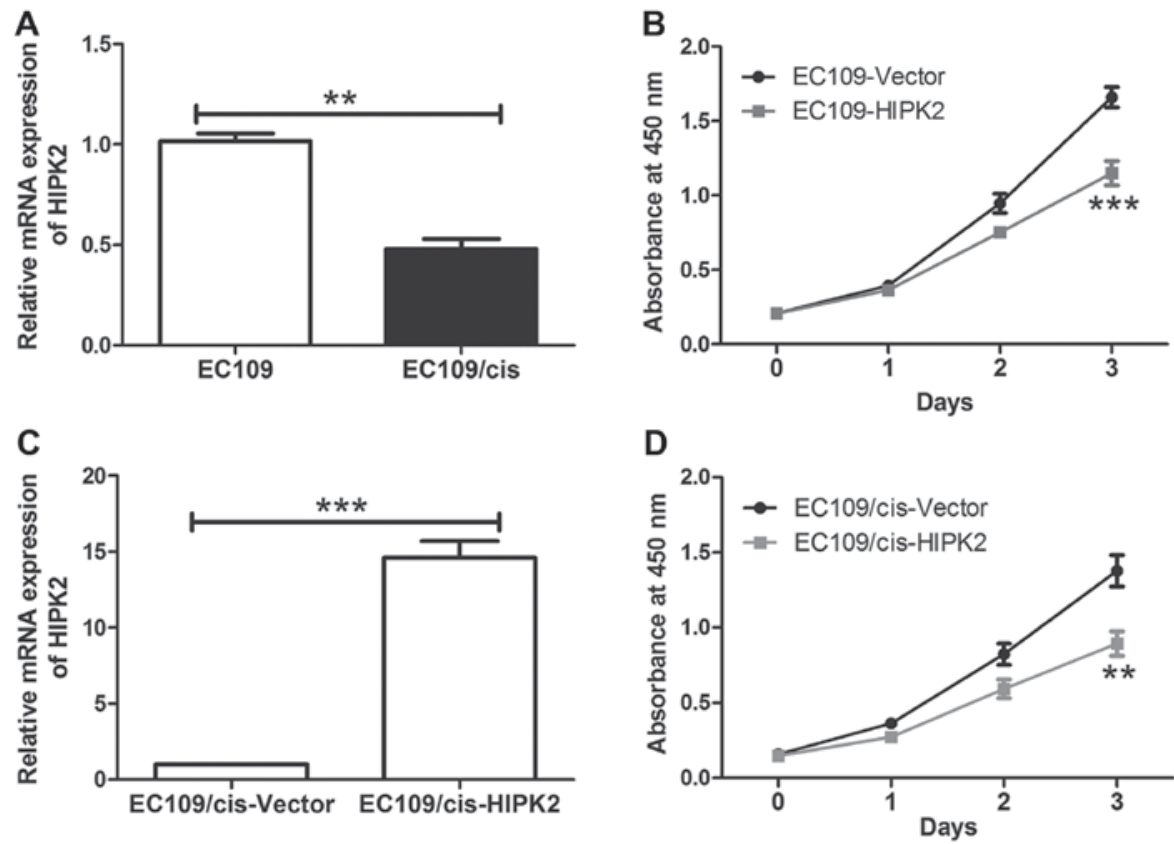

Figure 4. HIPK2 overexpression inhibits cell viability during cisplatin treatment. (A) Expression of HIPK2 was analyzed in EC109 and EC109/cis cells. (B) Proliferation analysis of EC109 cells transfected with HIPK2 and empty vector was analyzed by CCK-8 assay following cisplatin treatment (5 $\mu$ M). (C) HIPK2 expression was assessed by reverse transcription-quantitative polymerase chain reaction in EC109/cis cells transfected with HIPK2 and empty vector. (D) Proliferation analysis was performed using a CCK-8 assay following cisplatin treatment $(10 \mu \mathrm{M})$ in EC109/cis cells transfected with HIPK2 and empty vector. ${ }^{* * *} \mathrm{P}<0.01$ and ${ }^{* * * *} \mathrm{P}<0.001$. HIPK2, homeodomain-interacting protein kinase-2; cis, cisplatin; EC109/cis, chemo-resistant EC109 cells; CCK-8, Cell Counting Kit-8.

\section{Discussion}

The results of the present study indicate that HIPK2 is downregulated in ESCC tissues compared with their adjacent non-cancerous tissues. This result is consistent with previous studies, in which HIPK2 downregulation has been reported in bladder cancer, breast cancer and ovarian cancer $(12,14,22)$. HIPK2 overexpression has also been demonstrated in patients with cervical and colorectal cancer with familial adenomatous polyposis $(23,24)$. The current study demonstrated that the low expression of HIPK2 was associated with lymph node metastasis. These results suggest that HIPK 2 may be a candidate tumor suppressor associated with metastasis.

The role of lymphatic metastasis in aggressive malignancies has been demonstrated in multiple studies and the occurrence of regional lymph node metastasis at an early stage is a crucial step in cancer progression $(25,26)$. To investigate the effect of HIPK 2 on cell metastasis, stable HIPK2 overexpression and empty vector cell lines were established. Upregulation of HIPK2 inhibited the migration and invasion of ESCC cells. HIPK2 decreased the level of mesenchymal marker N-cadherin mRNA, which is one of the most important markers of EMT (27). By contrast, HIPK2 increased the expression of epithelial marker E-cadherin. The current study therefore demonstrates that HIPK2 suppresses EMT, which is an important step in cancer metastasis. Cell characteristics are altered during EMT, resulting in altered cell-cell and cell-matrix interactions, cell motility and invasiveness (28). Chung et al (29) previously reported that $\mathrm{N}$-cadherin suppresses $\mathrm{RAC}-\gamma$ serine/threonine-protein kinase to promote cell motility in mammary tumor cells. Furthermore, Qian et al (30) demonstrated that N-cadherin served a pivotal role in promoting metastasis through the regulation of extracellular-regulated kinase and protein kinase B. Nodale et al (14) demonstrated that MDA-MB-231 cells transfected with HIPK2 had decreased levels of vimentin mRNA that strongly correlated with re-expression of E-cadherin, which is indicative of a reversion of the EMT phenotype. Tan et al (15) also demonstrated that HIPK2 knockdown increased the levels of the mesenchymal markers $\mathrm{N}$-cadherin and fibronectin and decreased the level of E-cadherin, indicating that EMT is induced by HIPK2 downregulation. The results of the current study are consistent with previous studies.

Accumulating evidence also suggests that EMT is associated with the onset of drug resistance and tumor relapses, representing an escape mechanism from apoptosis (31). Systematic chemotherapy is an important clinical strategy used in the treatment of ESCC, which targets tumor cells and reduces tumor recurrence (32). It serves an important role in ESCC treatment, especially for patients with advanced and metastatic ESCC cancer (33). Acquired chemoresistance of a tumor leads to regrowth during anticancer agent treatment, even if the tumor initially responds to the anticancer agents (34). Lin et al (22) indicated that HIPK2 sensitized chemoresistant bladder cancer cells to cisplatin. In the present study, it was demonstrated that HIPK2 expression levels were significantly downregulated in EC109/cis cells compared with EC109 cells. The forced expression of HIPK2 reduced cell viability during cisplatin treatment in EC109 cells and HIPK2 overexpression ameliorated cisplatin resistance in EC109/cis cells. HIPK2 has been demonstrated to be an important regulator of $\mathrm{p} 53$ activity in response to chemotherapeutic agents (35). In addition, HIPK 2 overexpression in resensitized chemoresistant $\mathrm{p} 53$ wild type ovarian cancer cells to chemotherapy by mediating 
p53 phosphorylation (12). Consistent with previous studies, the present study demonstrated that HIPK 2 ameliorated chemoresistance, and this may be via regulation of p53 function, which will be investigated in the future.

In conclusion, the results of the current study demonstrate that HIPK2 is downregulated in ESCC specimens and is associated with metastasis. The results suggest that HIPK2 overexpression suppresses EMT and subsequent cell metastasis. Furthermore, HIPK2 overexpression partially ameliorates cisplatin resistance in EC109/cis cells. These results suggest that HIPK2 serves an important role in regulating the metastasis and chemoresistance of ESCC cells, suggesting a potential application of HIPK2 in ESCC therapy.

\section{References}

1. Wang LD, Zhou FY, Li XM, Sun LD, Song X, Jin Y, Li JM, Kong GQ, Qi H, Cui J, et al: Genome-wide association study of esophageal squamous cell carcinoma in Chinese subjects identifies susceptibility loci at PLCE1 and C20orf54. Nat Genet 42: 759-763, 2010.

2. Hongo M, Nagasaki Y and Shoji T: Epidemiology of esophageal cancer: Orient to Occident. Effects of chronology, geography and ethnicity. J Gastroenterol Hepatol 24: 729-735, 2009.

3. Morita M, Kumashiro R, Kubo N, Nakashima Y, Yoshida R, Yoshinaga K, Saeki H, Emi Y, Kakeji Y, Sakaguchi Y, et al: Alcohol drinking, cigarette smoking, and the development of squamous cell carcinoma of the esophagus: Epidemiology,clinical findings, and prevention. Int J Clin Oncol 15: 126-134, 2010.

4. Brown LM, Hoover R, Silverman D, Baris D, Hayes R, Swanson GM, Schoenberg J, Greenberg R, Liff J, Schwartz A, et al: Excess incidence of squamous cell esophagea cancer among US Black men: Role of social class and other risk factors. Am J Epidemiol 153: 114-122, 2001.

5. Pennathur A, Gibson MK, Jobe BA and Luketich JD: Oesophageal carcinoma. Lancet 381: 400-412, 2013.

6. Nardinocchi L, Puca R, Sacchi A, Rechavi G, Givol D and D'Orazi G: Targeting hypoxia in cancer cells by restoring homeodomain interacting protein-kinase 2 and p53 activity and suppressing HIF-1alpha. PLoS One 4: e6819, 2009.

7. Di Stefano V, Blandino G, Sacchi A, Soddu S and D'Orazi G: HIPK2 neutralizes MDM2 inhibition rescuing p53 transcriptional activity and apoptotic function. Oncogene 23: 5185-5192, 2004.

8. Bon G, Di Carlo SE, Folgiero V, Avetrani P, Lazzari C, D'Orazi G, Brizzi MF, Sacchi A, Soddu S, Blandino G, et al: Negative regulation of beta4 integrin transcription by homeodomain-interacting protein kinase 2 and p53 impairs tumor progression. Cancer Res 69: 5978-5986, 2009.

9. Ann EJ, Kim MY, Yoon JH, Ahn JS, Jo EH, Lee HJ, Lee HW, Kang HG, Choi DW, Chun KH, et al: Tumor suppressor HIPK2 regulates malignant growth via phosphorylation of Notch1. Cancer Res 76: 4728-4740, 2016.

10. Jin Y, Ratnam K, Chuang PY, Fan Y, Zhong Y, Dai Y, Mazloom AR, Chen EY, D'Agati V, Xiong H, et al: A systems approach identifies HIPK2 as a key regulator of kidney fibrosis. Nat Med 18: 580-588, 2012.

11. Pistritto G, Puca R, Nardinocchi L, Sacchi A and D'Orazi G: HIPK2-induced p53Ser46 phosphorylation activates the KILLER/DR5-mediated caspase-8 extrinsic apoptotic pathway. Cell Death Differ 14: 1837-1839, 2007.

12. Puca R, Nardinocchi L,Pistritto G and D'Orazi G: Overexpression of HIPK2 circumvents the blockade of apoptosis in chemoresistant ovarian cancer cells. Gynecol Oncol 109: 403-410, 2008

13. Lazzari C, Prodosmo A, Siepi F, Rinaldo C, Galli F, Gentileschi M, Bartolazzi A, Costanzo A, Sacchi A, Guerrini L and Soddu S: HIPK2 phosphorylates $\Delta \mathrm{Np} 63 \alpha$ and promotes its degradation in response to DNA damage. Oncogene 30: 4802-4813, 2011.

14. Nodale C, Sheffer M, Jacob-Hirsch J, Folgiero V, Falcioni R, Aiello A, Garufi A, Rechavi G, Givol D and D'Orazi G: HIPK2 downregulates vimentin and inhibits breast cancer cell invasion. Cancer Biol Ther 13: 198-205, 2012.
15. Tan M, Gong H, Zeng Y, Tao L, Wang J, Jiang J, Xu D, Bao E, Qiu J and Liu Z: Downregulation of homeodomain-interacting protein kinase-2 contributes to bladder cancer metastasis by regulating Wnt signaling. J Cell Biochem 115: 1762-1767, 2014.

16. Rice TW, Blackstone EH and Rusch VW: 7th edition of the AJCC cancer staging Manual: Esophagus and esophagogastric junction. Ann Surg Oncol 17: 1721-1724, 2010.

17. Han T, Zhu X, Wang J, Zhao H, Ma Q, Zhao J, Qiu X and Fan Q Establishment and characterization of a cisplatin-resistant human osteosarcoma cell line. Oncol Rep 32: 1133-1139, 2014.

18. Livak KJ and Schmittgen TD: Analysis of relative gene expression data using real-time quantitative PCR and the 2(-Delta Delta C(T)) method. Methods 25: 402-408, 2001

19. Zeisberg $M$ and Neilson EG: Biomarkers for epithelial-mesenchymal transitions. J Clin Invest 119: 1429-1437, 2009.

20. Akita H, Doki Y, Miyata H, Hirao T, Yano M, Takachi K, Miyashiro I, Sasaki Y, Ishikawa O, Ohigashi $\mathrm{H}$ and Imaoka S: Clinical significance of the second cycle response to cisplatin-based chemotherapy as preoperative treatment for esophageal squamous cell carcinoma. J Surg Oncol 93: 401-409, 2006.

21. Liu A, Zhu J, Wu G, Cao L, Tan Z, Zhang S, Jiang L, Wu J, Li M, Song L and Li J: Antagonizing miR-455-3p inhibits chemoresistance and aggressiveness in esophageal squamous cell carcinoma. Mol Cancer 16: 106, 2017

22. Lin J, Zhang Q, Lu Y, Xue W, Xu Y, Zhu Y and Hu X: Downregulation of HIPK2 increases resistance of bladder cancer cell to cisplatin by regulating Wip1. PLoS One 9: e98418, 2014.

23. Cheng Y, Al-Beiti MA, Wang J, Wei G, Li J, Liang S and Lu X: Correlation between homeodomain-interacting protein kinase 2 and apoptosis in cervical cancer. Mol Med Rep 5: 1251-1255, 2012.

24. D'Orazi G, Sciulli MG, Di Stefano V, Riccioni S, Frattini M, Falcioni R, Bertario L, Sacchi $A$ and Patrignani $P$ : Homeodomain-interacting protein kinase-2 restrains cytosolic phospholipase A2-dependent prostaglandin E2 generation in human colorectal cancer cells. Clin Cancer Res 12: 735-741, 2006.

25. Hsu WH, Hsu PK, Hsieh CC, Huang CS and Wu YC: The metastatic lymph node number and ratio are independent prognostic factors in esophageal cancer. J Gastrointest Surg 13: 1913-1920, 2009.

26. Hosch SB, Stoecklein NH, Pichlmeier U, Rehders A Scheunemann P, Niendorf A, Knoefel WT and Izbicki JR: Esophageal cancer: The mode of lymphatic tumor cell spread and its prognostic significance. J Clin Oncol 19: 1970-1975, 2001.

27. Shibue T and Weinberg RA: EMT, CSCs, and drug resistance: The mechanistic link and clinical implications. Nat Rev Clin Oncol 14: 611-629, 2017

28. Voulgari A and Pintzas A: Epithelial-mesenchymal transition in cancer metastasis: Mechanisms, markers and strategies to overcome drug resistance in the clinic. Biochim Biophys Acta 1796: 75-90, 2009.

29. Chung S, Yao J, Suyama K, Bajaj S, Qian X, Loudig OD, Eugenin EA, Phillips GR and Hazan RB: N-cadherin regulates mammary tumor cell migration through Akt3 suppression. Oncogene 32: 422-430, 2013.

30. Qian X, Anzovino A, Kim S, Suyama K, Yao J, Hulit J Agiostratidou G, Chandiramani N, McDaid HM, Nagi C, et al: N-cadherin/FGFR promotes metastasis through epithelial-to-mesenchymal transition and stem/progenitor cell-like properties. Oncogene 33: 3411-3421, 2014.

31. Mitra A, Mishra L and Li S: EMT, CTCs and CSCs in tumor relapse and drug-resistance. Oncotarget 6: 10697-10711, 2015.

32. Jin YY, Chen QJ, Xu K, Ren HT, Bao X, Ma YN, Wei Y and Ma HB: Involvement of microRNA-141-3p in 5-fluorouracil and oxaliplatin chemo-resistance in esophageal cancer cells via regulation of PTEN. Mol Cell Biochem 422: 161-170, 2016.

33. Lagergren J, Smyth E, Cunningham D and Lagergren P: Oesophageal cancer. Lancet: Jun 22, 2017 (Epub ahead of print).

34. Yoshida T, Miyoshi T, Seike J, Yamai H, Takechi H, Yuasa Y and Tangoku A: Gene expression changes in a chemoresistant model with human esophageal cancer xenografts using cDNA microarray. Anticancer Res 29: 1163-1168, 2009.

35. Puca R, Nardinocchi L, Givol D and D'Orazi G: Regulation of p53 activity by HIPK2: MOlecular mechanisms and therapeutical implications in human cancer cells. Oncogene 29: 4378-4387, 2010. 